\title{
Research
}

Dani Kim, Benedict Hayhoe, Paul Aylin, Martin R Cowie and Alex Bottle

\section{Health service use by patients with heart failure living in a community setting:}

\author{
a cross-sectional analysis in North West London
}

\begin{abstract}
\section{Background}

The complex nature of heart failure (HF) management, often involving multidimensional care, is widely recognised, but overall health service utilisation by patients with HF has not previously been described.
\end{abstract}

\section{Aim}

To describe overall health service use by adults with HF living in a community setting.

\section{Design and setting}

Cross-sectional analysis of prevalent HF cases from January 2015 to December 2018 using an administrative dataset covering primary and secondary care, and 'other' (community, mental health, social care) services in North West London

\section{Method}

Healthcare use of each service was described overall and by individual components of secondary care (such as, outpatient appointments), and 'other' services (such as, nursing contacts). Usage patterns were identified using $\mathrm{k}$-means cluster analysis, using all distinct contacts for the whole study period, and visualised with a heatmap.

\section{Results}

A total of 39301 patients with a prevalent diagnosis of HF between 1 January 2015 and 31 December 2018 were found. Of those, approximately $90 \%$ used health services during the study period, most commonly outpatient services, GP consultations, unplanned accident and emergency visits, and community services. Use of cardiology-specific services ranged from around 3\% (cardiology-related community care) to around $20 \%$ loutpatient cardiology visits). GP consultations decreased by $11 \%$ over the study period. Five clusters of patients were identified, each with statistically significantly different care usage patterns and patient characteristics.

\section{Conclusion}

Patients with HF make heavy but heterogeneous use of services. Relatively low and falling use of GP consultations, and the apparently low uptake of community rehabilitation services by patients with $\mathrm{HF}$, are concerning and suggest challenges in primary care access and integration of care.

\section{Keywords}

Heart failure; primary care; secondary care; outpatient services; cluster analysis; London.

\section{INTRODUCTION}

Heart failure (HF) affects $>900000$ people in the UK ${ }^{1}$ and results in significant morbidity and mortality, frequent hospitalisations, and reduced quality of life. Patients with HF are usually older with comorbidities, and may have complex and highly heterogeneous medical and social needs. A multidisciplinary team (MDT) approach is considered the gold standard model for $\mathrm{HF}$ management ${ }^{2}$ and is recommended for high-risk patients in the Health and Social Care Act of 2012,3 and other national ${ }^{4-6}$ and international guidelines. ${ }^{7.8}$ Despite this, there is currently little understanding of the nature of HF care beyond the hospital setting in the UK. Therefore, this study aimed to describe overall health and social service use and care usage patterns by patients with HF in North West London (NWL).

\section{METHOD}

\section{Data}

Whole Systems Integrated Care (WSIC) data were used: a linked de-identified dataset of individual-level patient records of events from primary, secondary, community, mental health, and social care services in NWL, covering $>2$ million patients across 400 GP practices. ${ }^{9.10}$ It has some similarities with primary care-based research databases like Clinical Practice Research Datalink (CPRD) and The Health Improvement Network (THIN), ${ }^{11,12}$ but with

D Kim, MSc, research assistant; B Hayhoe, PhD, MB, clinical lecturer in primary care; P Aylin, MB, FFPHM, professor of epidemiology and public health; A Bottle, PhD, professor of medical statistics, Dr Foster Unit, Department of Primary Care and Public Health, Imperial College London, London. MR Cowie, MB, MD, professor of cardiology, National Heart and Lung Institute, Imperial College London, London.

\section{Address for correspondence}

Alex Bottle, Department of Primary Care and Public Health, Imperial College London, Charing the addition of community, mental health, and social care service records.

\section{Definitions}

Patient characteristics. Sex, age, ethnicity, deprivation level, comorbidities, care status, blood pressure, body mass index (BMI), and smoking and alcohol drinking statuses were defined using primary care data at the start of each 1-year period, looking back 5 years to retrieve data. Socioeconomic status was based on the 2015 Index of Multiple Deprivation $(I M D)^{13}$ and divided into quintiles $(1=$ most deprived; $5=$ least deprived). Comorbidities were defined as per the Charlson Index in Khan et al, ${ }_{14}^{14}$ with some extra ones defined by the authors (see Supplementary Table S1 for details).

Health service use. Health service use was described for each cohort using data for that 1-year period. Primary care use was defined as having a consultation with a GP. Secondary care use included emergency admissions, elective admissions, unplanned accident and emergency (A\&E) visits, and outpatient visits. Use of 'other' health services (community, mental health, and social care services) were described overall and by individual components. Variables in these 'other' health service tables were often not in coded form, so contacts were first indexed with keywords (Box 1) arrived at iteratively by manually searching for the most common terms in each table.

Cross Campus, Reynolds Building, St Dunstan's Road, London W6 8RP, UK

Email: robert.bottledimperial.ac.uk

Submitted: 29 September 2019; Editor's response: 18 November 2019; final acceptance: 16 January 2020

\section{CBritish Journal of General Practice}

This is the full-length article (published online 16 Jun 2020) of an abridged version published in print. Cite this version as: $\mathbf{B r} \mathbf{J}$ Gen Pract 2020; DOI: https://doi.org/10.3399/bjgp20X711749 


\section{How this fits in}

Heart failure (HF) prevalence is increasing and requires multidisciplinary management, including within primary care. Using a linked database for North West London's 2.2 million population, this study found that in 39301 patients with $\mathrm{HF}$, only $60 \%$ had seen their GP and 20\% had been referred for cardiac rehabilitation during the study period, while overall use of unscheduled care by patients with $\mathrm{HF}$ was high, with $>40 \%$ using accident and emergency services. Findings from cluster analysis, highlighting groups of patients with $\mathrm{HF}$ that are particularly high and low users of elements of care, may facilitate active case finding and provision of more supportive and preventative care to improve outcomes for these patients.

\section{Cluster analysis}

The authors sought to discover patterns of healthcare utilisation via $\mathrm{k}$-means cluster analysis. ${ }^{15}$ Nine healthcare utilisation count variables, reflecting total usage from 2014 to 2018, were used to define clusters: emergency admissions; elective admissions; unplanned $A \& E$ visits not ending in admission; outpatient visits (cardiology); outpatient visits (other); GP consultations; and community, mental health, and social care contacts. Only distinct contacts and attended outpatient visits were included, and extreme high users (in the top $0.1 \%$ for any of these variables) were excluded.

Data were log-transformed and normalised (min-max method) before analysis to give higher weighting to lower values and equal weighting to all variables, respectively. K-means required the number of clusters ( $k=5)$ to be pre-specified (see Supplementary Box S1 for details).

\section{Statistical analysis}

Patient characteristics and prevalence of health service use were summarised for the four yearly cohorts and clusters separately. Usage patterns for each cluster were visualised using a heatmap by comparing the cluster mean usage with the average population usage, taking the percentage difference between these two means.

Differences in healthcare utilisation variables and key patient characteristics across clusters were analysed using Kruskal-Wallis tests for continuous variables and Pearson $\chi^{2}$ tests for categorical variables, with two-tailed testing and a significance level of 0.05. All analyses were conducted using R (version 3.4.0).

\section{RESULTS}

\section{Patient characteristics}

A total of 39301 patients from 359 GP practices between 1 January 2015 and 31 December 2018 had an HF diagnosis recorded and met the inclusion criteria (see Supplementary Figure S1 for flowchart of study population), that is, approximately 10 new patients with HF per practice per year. The vast majority of patients were in each of the four yearly cohorts.

In 2018, most patients were female $(56.2 \%, n=19463)$, aged $\geq 65$ years $(58.1 \%$, $n=20129)$, and were of white $(31.1 \%$, $n=10793$ ), Asian (25.7\%, $n=8905$ ), or unknown (27.3\%, $n=9454)$ ethnicity (Table 1). Almost two-thirds $163.8 \%$, $n=22$ 092) had multimorbidity, that is, had a comorbidity in addition to existing $\mathrm{HF}$, and of these more than half had at least two additional comorbidities, most commonly diabetes (26.1\%, $n=9053)$ or hypertension (36.1\%, $n=12$ 507).

A total of $6999(17.8 \%)$ people died and $110(0.3 \%)$ opted out of the WSIC dataset.

Most patient characteristics remained constant during the study period except for an increase in proportion of the underweight $160 \%$ increase) and the oldest age group (40\% increase), and a nearly $20 \%$ reduction in the prevalence of hypertension (Table 1).

\section{Health service use}

Approximately $90 \%$ of patients used health services during the study period Idata not shown). In 2018, the most commonly used healthcare services were outpatients (70.1\%, $n=24283$ ), GP consultations (59.9\%, $n=20$ 741), unplanned A\&E (40.8\%, $n=14$ 145), community (39.7\%, $n=13$ 762), emergency admissions $(26.7 \%, n=9257)$ and outpatient cardiology $(23.8 \%, n=8231)$ services (Table 2). Community care was the most common 'other' service used, of which the most frequent components were nursing (23.2\%, $n=8052)$, podiatry (15.6\%, $n=5397$ ), and rehabilitation-related services (8.3\%, $n=2861)$. Few $(2.9 \%$, $n=1005$ ) used community care related to cardiology even though $>1$ in 5 had a GP record of referral to cardiac rehabilitation. In total, $6.3 \%(n=2178)$ had a referral for echocardiogram, of which over half had abnormal results (51.1\%, $n=1113$ ). Both social care and mental health service use were less common $13.9 \%$ and $4.5 \%$, respectively). When used, mental health contacts were commonly communityrelated $(4.3 \%, n=1489)$, suggesting a community integrated approach; social care contacts were personal care $13.1 \%$, $n=1057)$, community $(0.7 \%, \quad n=232)$, 


\section{Box 1. Key terms used to index individual components in 'other' services to describe the types of services used by patients with HF in respective settings}

\begin{tabular}{|c|c|}
\hline Service and component & Index terms \\
\hline \multicolumn{2}{|l|}{ Community } \\
\hline Nursing & nursing \\
\hline Rehabilitation & rehaba \\
\hline Urgent & $\begin{array}{l}\text { rapid, acute, urgent, emergency, } A \& E \text {, unplanned, admit }{ }^{a} \text {, hospital } \\
\text { admª inpatient, ambulance }\end{array}$ \\
\hline Intermediate & intermediate, CIS \\
\hline Cardiology & heart, cardia, stroke \\
\hline Diabetes & diabet $^{a}$, endocri ${ }^{a}$ \\
\hline Physio- or occupational therapy & occupation ${ }^{a}$, physio $^{a}$ \\
\hline Podiatry & foot, poda \\
\hline Respiratory & pulmon ${ }^{a}$, respira, COPD, TB, tubercula, thorac ${ }^{a}$ \\
\hline Neurology & musculo ${ }^{a}, M S K$, neuro ${ }^{a}$, parkinson ${ }^{a}$ \\
\hline Urinary & genito $^{a}$, bladder, bowel, continenc ${ }^{a}$, urinary \\
\hline Speech language therapy & SLT, speech, language \\
\hline Falls & falls \\
\hline Diet and nutrition & diet, nutrition ${ }^{a}$ \\
\hline Memory and cognition & memory, cognition \\
\hline Home & home \\
\hline Phone & phone \\
\hline Unknown & (none of the above key terms) \\
\hline \multicolumn{2}{|l|}{ Mental health } \\
\hline Outpatient & outpatient, day case \\
\hline Community & community \\
\hline Urgent & rapid, acute, urgent, emergency, $A \& E$, unplanned \\
\hline Specialist & special ${ }^{a}$, nurs $^{a},{ }^{a}$ ist \\
\hline Dementia & dementi ${ }^{a}$, memory, cogni $i^{a}$ \\
\hline Learning disability & learning \\
\hline Eating disorder & eating, anorexia \\
\hline Psychiatric & $p s y^{a}$ \\
\hline Review & review ${ }^{a}$ \\
\hline Consultation & consulta \\
\hline Treatment & treat $^{a}$ \\
\hline Assessment & assess $^{a}$ \\
\hline Unknown & (none of the above key terms) \\
\hline \multicolumn{2}{|l|}{ Social care } \\
\hline Nursing & nursing \\
\hline Rehabilitation & rehaba \\
\hline Urgent & rapid, acute, urgent, emergency, $A \& E$, unplanned \\
\hline Personal care & $\begin{array}{l}\text { personal care, home care, day care, bathing, extra care, reablea, care } \\
\text { service }\end{array}$ \\
\hline Food & food, meal \\
\hline Domestic & $\begin{array}{l}\text { domiciliary, domestic, housework, laundry, shopping, cleaning, wash- } \\
\text { ing }\end{array}$ \\
\hline Transport & transfer, transport, migration, trip, mobil ${ }^{a}$ \\
\hline Disability & disy, disabilita ${ }^{2}$ disable \\
\hline Occupational therapy & occupational therapy \\
\hline Memory and cognition & dementia, memory, cognition \\
\hline Assisted equipment technology & assistive techa, assisted equipment, equipment, technology \\
\hline Nursing home & nursing home, residential home, residential care, care home \\
\hline Mental health & mental, CMHT \\
\hline Community & community \\
\hline Social & social \\
\hline Carer & carer \\
\hline Housing and living & housing, living \\
\hline Unknown & (none of the above key terms) ${ }^{a}$ \\
\hline
\end{tabular}

aSuperscript used as a wildcard character during key term search. When used, search returns results containing text preceding ${ }^{\prime}$. $A \& E=$ accident and emergency. $C / S=$ community independence service. $C M H T=$ community mental health team. $C O P D=$ chronic obstructive pulmonary disease. $H F=$ heart failure. MSK = musculoskeletal. $S L T=$ speech language therapy. $T B=$ tuberculosis. domestic $(0.7 \%, n=253)$, and disabilityrelated $(0.7 \%, n=248)$ (Table 2$)$.

In 2018, only 3067 (8.9\%) patients did not use any services, while around onequarter used $>3$ different types $124.8 \%$, $n=8607)$. Services were most commonly used in combination with secondary care and least commonly with 'other' health services (Table 2 and Figure 1). Few patients used only primary care and 'other' services $(2.3 \%, n=781)$ or 'other' services alone $(2.0 \%, n=682)$

Over the study period, health service use increased for all elements of secondary care analysed, particularly elective admissions (37\% increase) and outpatient visits (24\% increase), but decreased for primary care (11\% decrease). Though many components of the community contacts remained constant, there were more than double contacts related to diet and nutrition (Table 2).

\section{Cluster analysis}

Altogether 318 patients were excluded from the k-means cluster analysis due to extremely high usage. Of the four and fivecluster solutions identified via preliminary analysis (see Supplementary Box S1), the five-cluster solution was chosen as the extra cluster had distinct usage patterns (Figure 2). Additionally, all patient characteristics differed significantly across clusters (see Supplementary Table S2 and Supplementary Figure S2). Patients who were younger, female, with less comorbidity, and not living in care homes were generally low users of health care (clusters 1 and 2). Perhaps unsurprisingly, those with higher blood pressure and more comorbidities had relatively more GP consultations (cluster 2). Patients who were older, male, and had more comorbidities were generally higher users of health care (clusters 3, 4 and 5). The lowest users of GP appointments were very high users of all other services (cluster 3, Figure 2). Those with the most cardiovascular comorbidity (cluster 4) had the highest usage of cardiology-related outpatient services and referrals to echocardiography (42.0\%) (Figure 2). The oldest patients with the highest mortality (cluster 5) were the highest users of emergency inpatient, A\&E, and 'other' services (Figure 2).

\section{DISCUSSION}

\section{Summary}

Overall health service utilisation was high. Almost everyone in the present study population used some kind of health service during the study period: outpatients (7 in 10), 
Table 1. Patient characteristics for each cohort

\begin{tabular}{|c|c|c|c|c|c|c|c|c|}
\hline \multirow[b]{3}{*}{ Characteristics } & \multicolumn{8}{|c|}{ Year } \\
\hline & \multicolumn{2}{|c|}{2015} & \multicolumn{2}{|c|}{2016} & \multicolumn{2}{|l|}{2017} & \multicolumn{2}{|c|}{2018} \\
\hline & $n(N=23828)$ & $\%$ & $n(N=27443)$ & $\%$ & $n(N=31554)$ & $\%$ & $n(N=34651)$ & $\%$ \\
\hline \multicolumn{9}{|l|}{ Sex } \\
\hline Female & 13178 & 55.3 & 15246 & 55.6 & 17506 & 55.5 & 19463 & 56.2 \\
\hline \multicolumn{9}{|l|}{ Age group, years } \\
\hline$<45$ & 3293 & 13.8 & 3958 & 14.4 & 4619 & 14.6 & 5227 & 15.1 \\
\hline 45 to $<65$ & 6760 & 28.4 & 7661 & 27.9 & 8600 & 27.3 & 9295 & 26.8 \\
\hline 65 to $<75$ & 5542 & 23.3 & 5957 & 21.7 & 6601 & 20.9 & 7007 & 20.2 \\
\hline 75 to $<85$ & 6002 & 25.2 & 6952 & 25.3 & 7948 & 25.2 & 8470 & 24.4 \\
\hline$>85$ & 2231 & 9.4 & 2915 & 10.6 & 3786 & 12.0 & 4652 & 13.4 \\
\hline \multicolumn{9}{|c|}{ Deprivation level, IMD } \\
\hline 1 (most) & 3166 & 13.3 & 3883 & 14.1 & 4656 & 14.8 & 5205 & 15.0 \\
\hline 2 & 6568 & 27.6 & 7673 & 28.0 & 9001 & 28.5 & 9954 & 28.7 \\
\hline 3 & 5869 & 24.6 & 6833 & 24.9 & 7875 & 25.0 & 8759 & 25.3 \\
\hline 4 & 4089 & 17.2 & 4596 & 16.7 & 5197 & 16.5 & 5653 & 16.3 \\
\hline 5 (least) & 3430 & 14.4 & 3636 & 13.2 & 3866 & 12.3 & 3975 & 11.5 \\
\hline Unknown & 706 & 3.0 & 822 & 3.0 & 959 & 3.0 & 1105 & 3.2 \\
\hline \multicolumn{9}{|l|}{ Ethnicity } \\
\hline White & 7712 & 32.4 & 8741 & 31.9 & 9963 & 31.6 & 10793 & 31.1 \\
\hline Asian & 6237 & 26.2 & 7147 & 26.0 & 8161 & 25.9 & 8905 & 25.7 \\
\hline Black & 1721 & 7.2 & 1990 & 7.3 & 2245 & 7.1 & 2412 & 7.0 \\
\hline Mixed & 1767 & 7.4 & 2162 & 7.9 & 2630 & 8.3 & 3087 & 8.9 \\
\hline Unknown & 6391 & 26.8 & 7403 & 27.0 & 8555 & 27.1 & 9454 & 27.3 \\
\hline \multicolumn{9}{|l|}{ Care status } \\
\hline Care home & 619 & 2.6 & 682 & 2.5 & 732 & 2.3 & 686 & 2.0 \\
\hline Have carer & 125 & 0.5 & 162 & 0.6 & 191 & 0.6 & 219 & 0.6 \\
\hline \multicolumn{9}{|l|}{ Status } \\
\hline Died & 1294 & 5.4 & 1543 & 5.6 & 1811 & 5.7 & 2351 & 6.8 \\
\hline Opted out & - & 0.0 & 21 & 0.1 & 92 & 0.3 & 5 & 0.0 \\
\hline \multicolumn{9}{|l|}{ Smoking status } \\
\hline Non & 9612 & 40.3 & 11455 & 41.7 & 13395 & 42.5 & 14955 & 43.2 \\
\hline Current & 2290 & 9.6 & 2648 & 9.6 & 3174 & 10.1 & 3377 & 9.7 \\
\hline Former & 7626 & 32.0 & 8390 & 30.6 & 9267 & 29.4 & 9685 & 28.0 \\
\hline Unknown & 4300 & 18.0 & 4950 & 18.0 & 5718 & 18.1 & 6634 & 19.1 \\
\hline \multicolumn{9}{|l|}{ Drinking status } \\
\hline Non & 1607 & 6.7 & 1934 & 7.0 & 2079 & 6.6 & 2197 & 6.3 \\
\hline Current & 382 & 1.6 & 439 & 1.6 & 485 & 1.5 & 578 & 1.7 \\
\hline Former & 43 & 0.2 & 58 & 0.2 & 70 & 0.2 & 83 & 0.2 \\
\hline Unknown & 21796 & 91.5 & 25012 & 91.1 & 28920 & 91.7 & 31793 & 91.8 \\
\hline \multicolumn{9}{|l|}{ BMI category } \\
\hline Underweight & 423 & 1.8 & 526 & 1.9 & 714 & 2.3 & 1000 & 2.9 \\
\hline Ideal & 5210 & 21.9 & 6000 & 21.9 & 7032 & 22.3 & 7814 & 22.6 \\
\hline Overweight & 6296 & 26.4 & 7089 & 25.8 & 7959 & 25.2 & 8503 & 24.5 \\
\hline Obese & 6255 & 26.3 & 7213 & 26.3 & 8323 & 26.4 & 8891 & 25.7 \\
\hline Unknown & 5644 & 23.7 & 6615 & 24.1 & 7526 & 23.9 & 8443 & 24.4 \\
\hline \multicolumn{9}{|c|}{ Systolic blood pressure, $\mathrm{mm} \mathrm{Hg}$} \\
\hline$<110$ & 1109 & 4.7 & 1303 & 4.7 & 1535 & 4.9 & 1789 & 5.2 \\
\hline $110-119$ & 2386 & 10.0 & 2886 & 10.5 & 3497 & 11.1 & 3966 & 11.4 \\
\hline 120-139 & 10943 & 45.9 & 12768 & 46.5 & 14820 & 47.0 & 16465 & 47.5 \\
\hline $140-159$ & 5925 & 24.9 & 6540 & 23.8 & 7270 & 23.0 & 7623 & 22.0 \\
\hline$>159$ & 510 & 2.1 & 570 & 2.1 & 627 & 2.0 & 609 & 1.8 \\
\hline Unknown & 2955 & 12.4 & 3376 & 12.3 & 3805 & 12.1 & 4199 & 12.1 \\
\hline
\end{tabular}

primary care ( 6 in 10 saw a GP), community services, especially nursing (2 in 10), and unplanned $A \& E$ visits ( 4 in 10). Community care use related to cardiology was low. Few patients used only primary care and 'other' services, which may reflect modest needs or a lack of community and primary care provision suitable for complex needs.

Patterns of health service utilisation depended on age and comorbidity but were 
Table 1 Continued. Patient characteristics for each cohort

\begin{tabular}{|c|c|c|c|c|c|c|c|c|}
\hline \multirow[b]{3}{*}{ Characteristics } & \multicolumn{8}{|c|}{ Year } \\
\hline & \multicolumn{2}{|l|}{2015} & \multicolumn{2}{|c|}{2016} & \multicolumn{2}{|c|}{2017} & \multicolumn{2}{|c|}{2018} \\
\hline & $n(N=23828)$ & $\%$ & $n(N=27443)$ & $\%$ & $n(N=31554)$ & $\%$ & $n(N=34651)$ & $\%$ \\
\hline$<80$ & 13969 & 58.6 & 16434 & 59.9 & 19215 & 60.9 & 21261 & 61.4 \\
\hline 80 to 89 & 5586 & 23.4 & 6195 & 22.6 & 6959 & 22.1 & 7536 & 21.7 \\
\hline 90 to 99 & 962 & 4.0 & 1053 & 3.8 & 1159 & 3.7 & 1219 & 3.5 \\
\hline$>99$ & 134 & 0.6 & 148 & 0.5 & 166 & 0.5 & 179 & 0.5 \\
\hline Unknown & 3177 & 13.3 & 3613 & 13.2 & 4055 & 12.9 & 4456 & 12.9 \\
\hline \multicolumn{9}{|l|}{ Comorbidities } \\
\hline Acute myocardial infarction & 1006 & 4.2 & 1123 & 4.1 & 1309 & 4.1 & 1398 & 4.0 \\
\hline Atrial fibrillation & 2700 & 11.3 & 3362 & 12.3 & 4018 & 12.7 & 4549 & 13.1 \\
\hline Chronic pulmonary disease & 3346 & 14.0 & 3928 & 14.3 & 4623 & 14.7 & 5084 & 14.7 \\
\hline Congenital heart disease & 73 & 0.3 & 91 & 0.3 & 122 & 0.4 & 135 & 0.4 \\
\hline Coronary heart disease & 1516 & 6.4 & 1658 & 6.0 & 1856 & 5.9 & 1965 & 5.7 \\
\hline Myocardial infarction & 1034 & 4.3 & 1146 & 4.2 & 1331 & 4.2 & 1444 & 4.2 \\
\hline Myocarditis & 614 & 2.6 & 697 & 2.5 & 797 & 2.5 & 868 & 2.5 \\
\hline Other arrhythmias & 2181 & 9.2 & 2680 & 9.8 & 3266 & 10.4 & 3782 & 10.9 \\
\hline Peripheral vascular disease & 563 & 2.4 & 634 & 2.3 & 681 & 2.2 & 771 & 2.2 \\
\hline Renal diseases & 1048 & 4.4 & 1129 & 4.1 & 1301 & 4.1 & 1400 & 4.0 \\
\hline Stroke & 667 & 2.8 & 762 & 2.8 & 933 & 3.0 & 1052 & 3.0 \\
\hline \multicolumn{9}{|l|}{ Comorbidities, $n$} \\
\hline 0 & 7793 & 32.7 & 9329 & 34.0 & 11073 & 35.1 & 12559 & 36.2 \\
\hline 1 & 7155 & 30.0 & 7978 & 29.1 & 8950 & 28.4 & 9667 & 27.9 \\
\hline 2 & 4976 & 20.9 & 5640 & 20.6 & 6264 & 19.9 & 6659 & 19.2 \\
\hline 3 & 2291 & 9.6 & 2632 & 9.6 & 3082 & 9.8 & 3363 & 9.7 \\
\hline$\geq 4$ & 1613 & 6.8 & 1864 & 6.8 & 2185 & 6.9 & 2403 & 6.9 \\
\hline
\end{tabular}

Figure 1. Venn diagram of service use in 2018, showing an approximation of group sizes. An intersection is missing between primary care and 'other' services (2.3\%) - documentation of $R$ eulerr package states with three or more sets intersecting, exact Euler diagrams are often impossible. For such cases eulerr attempts to provide a good approximation. ${ }^{16}$ highly heterogeneous. Younger patients with fewer comorbidities (clusters 1 and 2) had the lowest usage, which may partly indicate underutilisation and/or lack of access. For instance, those with infrequent GP consultations (clusters 1 and 3) were also more likely to be of mixed ethnicity and living in areas of higher deprivation and demographics known to be associated with poorer primary care access. ${ }^{17,18}$ These patients also showed the highest levels of unknown values for patient variables (derived from GP datal and lower than average GP consultation rates, which could reflect both poor health management and low engagement of patients in their

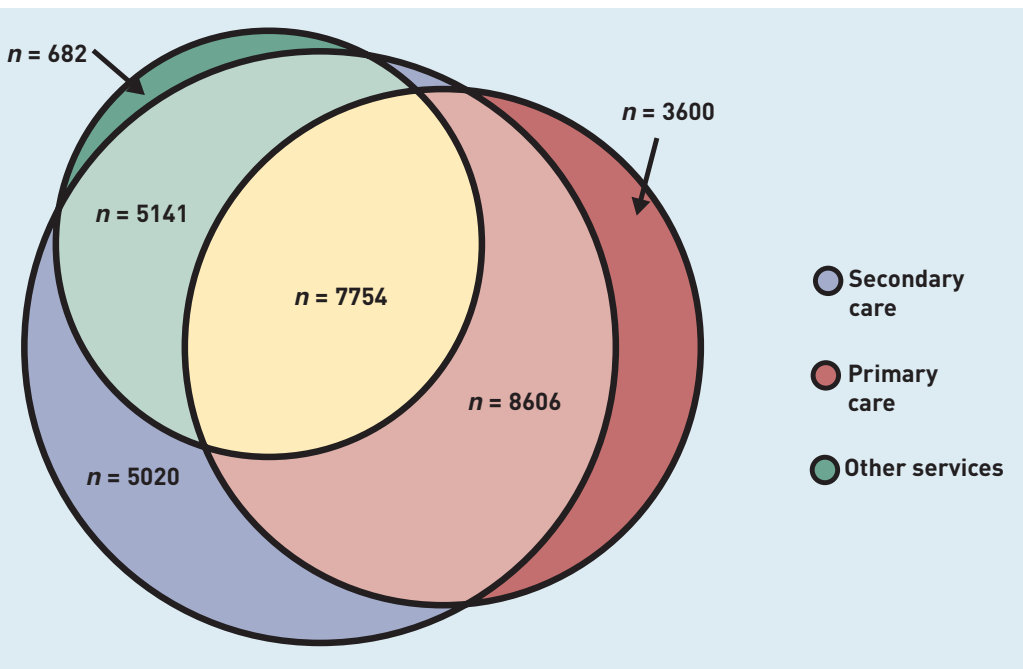


Table 2. Health service ever used by patients with HF in NWL between 2015 and 2018

\begin{tabular}{|c|c|c|c|c|c|c|c|c|}
\hline \multirow[b]{3}{*}{ Service } & \multicolumn{8}{|c|}{ Year } \\
\hline & \multicolumn{2}{|c|}{2015} & \multicolumn{2}{|l|}{2016} & \multicolumn{2}{|c|}{2017} & \multicolumn{2}{|c|}{2018} \\
\hline & $n(N=23828)$ & $\%$ & $n(N=27443)$ & $\%$ & $n(N=31554)$ & $\%$ & $n(N=34651)$ & $\%$ \\
\hline \multicolumn{9}{|l|}{ Secondary care } \\
\hline Emergency admission & 5163 & 21.7 & 6592 & 24.0 & 8276 & 26.2 & 9257 & 26.7 \\
\hline Elective admission & 910 & 3.8 & 1495 & 5.4 & 1803 & 5.7 & 1798 & 5.2 \\
\hline Unplanned A\&E & 8238 & 34.6 & 10697 & 39.0 & 12250 & 38.8 & 14145 & 40.8 \\
\hline Outpatient (any specialty) & 13492 & 56.6 & 18560 & 67.6 & 22115 & 70.1 & 24283 & 70.1 \\
\hline \multicolumn{9}{|l|}{ Outpatient (top 1) } \\
\hline (cardiology) & 4117 & 17.3 & 6518 & 23.8 & 8040 & 25.5 & 8231 & 23.8 \\
\hline Outpatient (top 2) & 2645 & 11.1 & 4662 & 17.0 & 5600 & 17.7 & 6049 & 17.5 \\
\hline Outpatient (top 3) ${ }^{\mathrm{a}}$ & 1992 & 8.4 & 3444 & 12.5 & 4127 & 13.1 & 5053 & 14.6 \\
\hline Outpatient (top 4) & 1645 & 6.9 & 3156 & 11.5 & 4018 & 12.7 & 4500 & 13.0 \\
\hline Outpatient (top 5) & 1563 & 6.6 & 2554 & 9.3 & 3220 & 10.2 & 3383 & 9.8 \\
\hline \multicolumn{9}{|l|}{ Primary care } \\
\hline GP consultation & 16014 & 67.2 & 17573 & 64.0 & 19494 & 61.8 & 20741 & 59.9 \\
\hline Echocardiogram abnormal & 1010 & 4.2 & 1066 & 3.9 & 1150 & 3.6 & 1113 & 3.2 \\
\hline \multicolumn{9}{|l|}{ Community } \\
\hline Any & 8801 & 36.9 & 9869 & 36.0 & 11195 & 35.5 & 13762 & 39.7 \\
\hline Nursing & 6590 & 27.7 & 5893 & 21.5 & 6311 & 20.0 & 8052 & 23.2 \\
\hline Rehabilitation & 2052 & 8.6 & 2489 & 9.1 & 2065 & 6.5 & 2861 & 8.3 \\
\hline Urgent & 918 & 3.9 & 811 & 3.0 & 999 & 3.2 & 1203 & 3.5 \\
\hline Intermediate & 1841 & 7.7 & 1754 & 6.4 & 1397 & 4.4 & 1526 & 4.4 \\
\hline Cardiology & 859 & 3.6 & 578 & 2.1 & 797 & 2.5 & 1005 & 2.9 \\
\hline \multicolumn{9}{|l|}{ GP referral to cardiac } \\
\hline rehabilitation & 4333 & 18.2 & 5465 & 19.9 & 6585 & 20.9 & 7187 & 20.7 \\
\hline Diabetes & 1011 & 4.2 & 955 & 3.5 & 1171 & 3.7 & 1654 & 4.8 \\
\hline \multicolumn{9}{|l|}{ Physio-/occupational } \\
\hline therapy & 1091 & 4.6 & 1160 & 4.2 & 1148 & 3.6 & 1853 & 5.3 \\
\hline Podiatry & 3760 & 15.8 & 4076 & 14.9 & 4404 & 14.0 & 5397 & 15.6 \\
\hline Respiratory & 217 & 0.9 & 163 & 0.6 & 206 & 0.7 & 302 & 0.9 \\
\hline Speech language therapy & 108 & 0.5 & 68 & 0.2 & 60 & 0.2 & 102 & 0.3 \\
\hline Falls & 165 & 0.7 & 332 & 1.2 & 365 & 1.2 & 450 & 1.3 \\
\hline Diet and nutrition & 644 & 2.7 & 1399 & 5.1 & 1696 & 5.4 & 2197 & 6.3 \\
\hline Home & 1229 & 5.2 & 1411 & 5.1 & 1738 & 5.5 & 1654 & 4.8 \\
\hline Phone & 949 & 4.0 & 317 & 1.2 & 418 & 1.3 & 393 & 1.1 \\
\hline Unknown & 178 & 0.7 & 203 & 0.7 & 206 & 0.7 & 248 & 0.7 \\
\hline \multicolumn{9}{|l|}{ Mental health } \\
\hline Any & 1064 & 4.5 & 1421 & 5.2 & 1718 & 5.4 & 1557 & 4.5 \\
\hline Outpatient & 354 & 1.5 & 360 & 1.3 & 428 & 1.4 & 169 & 0.5 \\
\hline Community & 964 & 4.0 & 1305 & 4.8 & 1589 & 5.0 & 1489 & 4.3 \\
\hline Urgent & 387 & 1.6 & 695 & 2.5 & 950 & 3.0 & 631 & 1.8 \\
\hline Specialist & 62 & 0.3 & 197 & 0.7 & 253 & 0.8 & 78 & 0.2 \\
\hline Dementia & 527 & 2.2 & 585 & 2.1 & 706 & 2.2 & 732 & 2.1 \\
\hline Learning disability & 33 & 0.1 & 39 & 0.1 & 48 & 0.2 & 29 & 0.1 \\
\hline Psychology & 91 & 0.4 & 236 & 0.9 & 279 & 0.9 & 261 & 0.8 \\
\hline Review & 109 & 0.5 & 159 & 0.6 & 214 & 0.7 & 92 & 0.3 \\
\hline Consultation & 51 & 0.2 & 82 & 0.3 & 118 & 0.4 & 951 & 2.7 \\
\hline Treatment & 61 & 0.3 & 84 & 0.3 & 110 & 0.3 & 117 & 0.3 \\
\hline Assessment & 151 & 0.6 & 254 & 0.9 & 309 & 1.0 & 267 & 0.8 \\
\hline Unknown & $<5$ & 0.0 & $<5$ & 0.0 & - & 0.0 & - & 0.0 \\
\hline
\end{tabular}

own health (they were also more likely to be smokers). The oldest and most likely to live in care homes (cluster 5) had the highest usage of emergency inpatient, $A \& E$, and 'other' services, and had high levels of comorbidity, especially renal disease, and the highest mortality. Higher usage of care is expected in older patients with 
Table 2 Continued. Health service ever used by patients with HF in NWL between 2015 and 2018

\begin{tabular}{|c|c|c|c|c|c|c|c|c|}
\hline \multirow[b]{3}{*}{ Service } & \multicolumn{8}{|c|}{ Year } \\
\hline & \multicolumn{2}{|c|}{2015} & \multicolumn{2}{|l|}{2016} & \multicolumn{2}{|l|}{2017} & \multicolumn{2}{|c|}{2018} \\
\hline & $n(N=23828)$ & $\%$ & $n(N=27443)$ & $\%$ & $n(N=31554)$ & $\%$ & $n(N=34651)$ & $\%$ \\
\hline Any & 1042 & 4.4 & 1236 & 4.5 & 1912 & 6.1 & 1350 & 3.9 \\
\hline Nursing & 89 & 0.4 & 109 & 0.4 & 169 & 0.5 & 128 & 0.4 \\
\hline Personal care & 709 & 3.0 & 890 & 3.2 & 1485 & 4.7 & 1057 & 3.1 \\
\hline Food & 68 & 0.3 & 82 & 0.3 & 44 & 0.1 & 10 & 0.0 \\
\hline Domestic & 165 & 0.7 & 238 & 0.9 & 350 & 1.1 & 253 & 0.7 \\
\hline Transport & 78 & 0.3 & 78 & 0.3 & 99 & 0.3 & 70 & 0.2 \\
\hline Disability & 181 & 0.8 & 230 & 0.8 & 277 & 0.9 & 248 & 0.7 \\
\hline Occupational therapy & 8 & 0.0 & 15 & 0.1 & 18 & 0.1 & 28 & 0.1 \\
\hline Memory and cognition & 53 & 0.2 & 76 & 0.3 & 104 & 0.3 & 63 & 0.2 \\
\hline \multicolumn{9}{|l|}{ Assisted equipment } \\
\hline technology & 129 & 0.5 & 28 & 0.1 & 47 & 0.1 & 7 & 0.0 \\
\hline Nursing home & 58 & 0.2 & 76 & 0.3 & 123 & 0.4 & 80 & 0.2 \\
\hline Community & 250 & 1.0 & 122 & 0.4 & 198 & 0.6 & 232 & 0.7 \\
\hline Social & 64 & 0.3 & 87 & 0.3 & 165 & 0.5 & 88 & 0.3 \\
\hline Carer & 127 & 0.5 & 125 & 0.5 & 142 & 0.5 & 159 & 0.5 \\
\hline Housing and living & 119 & 0.5 & 66 & 0.2 & 98 & 0.3 & 41 & 0.1 \\
\hline Unknown & 68 & 0.3 & 110 & 0.4 & 119 & 0.4 & 66 & 0.2 \\
\hline \multicolumn{9}{|l|}{ Number of services used } \\
\hline 0 & 2602 & 10.9 & 2567 & 9.4 & 2891 & 9.2 & 3067 & 8.9 \\
\hline 1 & 7036 & 29.5 & 7438 & 27.1 & 8572 & 27.2 & 9267 & 26.7 \\
\hline 2 & 8200 & 34.4 & 10549 & 38.4 & 12211 & 38.7 & 13710 & 39.6 \\
\hline$>3$ & 5990 & 25.1 & 6889 & 25.1 & 7880 & 24.9 & 8607 & 24.8 \\
\hline \multicolumn{9}{|l|}{ Service type } \\
\hline None & 2602 & 10.9 & 2567 & 9.4 & 2891 & 9.2 & 3067 & 8.9 \\
\hline Secondary care only & 2508 & 10.5 & 3569 & 13.0 & 4586 & 14.5 & 5020 & 14.5 \\
\hline Primary care only & 4134 & 17.3 & 3443 & 12.5 & 3538 & 11.2 & 3600 & 10.4 \\
\hline Other services only & 460 & 1.9 & 467 & 1.7 & 486 & 1.5 & 682 & 2.0 \\
\hline \multicolumn{9}{|l|}{ Secondary care } \\
\hline and other services & 2244 & 9.4 & 3267 & 11.9 & 4097 & 13.0 & 5141 & 14.8 \\
\hline \multicolumn{9}{|l|}{ Primary care } \\
\hline and other services & 1133 & 4.8 & 606 & 2.2 & 614 & 1.9 & 781 & 2.3 \\
\hline All three & 5449 & 22.9 & 6193 & 22.6 & 6877 & 21.8 & 7754 & 22.4 \\
\hline
\end{tabular}

aTop five outpatient specialties by year: 2015 ranking: 1) cardiology; 2) general surgery; 3) ophthalmology; 4) trauma and orthopaedics; 5) allied health professional episode. 2016 to 2018 ranking: 1) cardiology; 2) ophthalmology; 3) general surgery; 4) allied health professional episode; 5) radiology. A\&E = accident and emergency department. $H F=$ heart failure. $N W L=$ North West London .

comorbidities, ${ }^{19-21}$ but some use might be excessive and avoidable. ${ }^{19,20}$ Health service use was high in the present population of adults with HF living in a community setting. However, overall, relatively low GP service use, which decreased over the study period, and high use of emergency and other unscheduled care in these vulnerable patients is of significant concern and may suggest challenges in access to primary care services. These findings warrant further investigation to ensure equity of access and appropriate integrated care provision for patients with $\mathrm{HF}$.

\section{Strengths and limitations}

The authors used a linked dataset with nearcomplete population coverage for the region and employed both descriptive analysis and clustering algorithms to describe health service use by this highly heterogeneous population. The dataset is large and reflective of current medical practice, but the study has several limitations.

Electronic health records are not specifically intended for research, and coding is highly variable. ${ }^{22}$ Coding in some of the WSIC tables required additional cleaning and processing, which could 


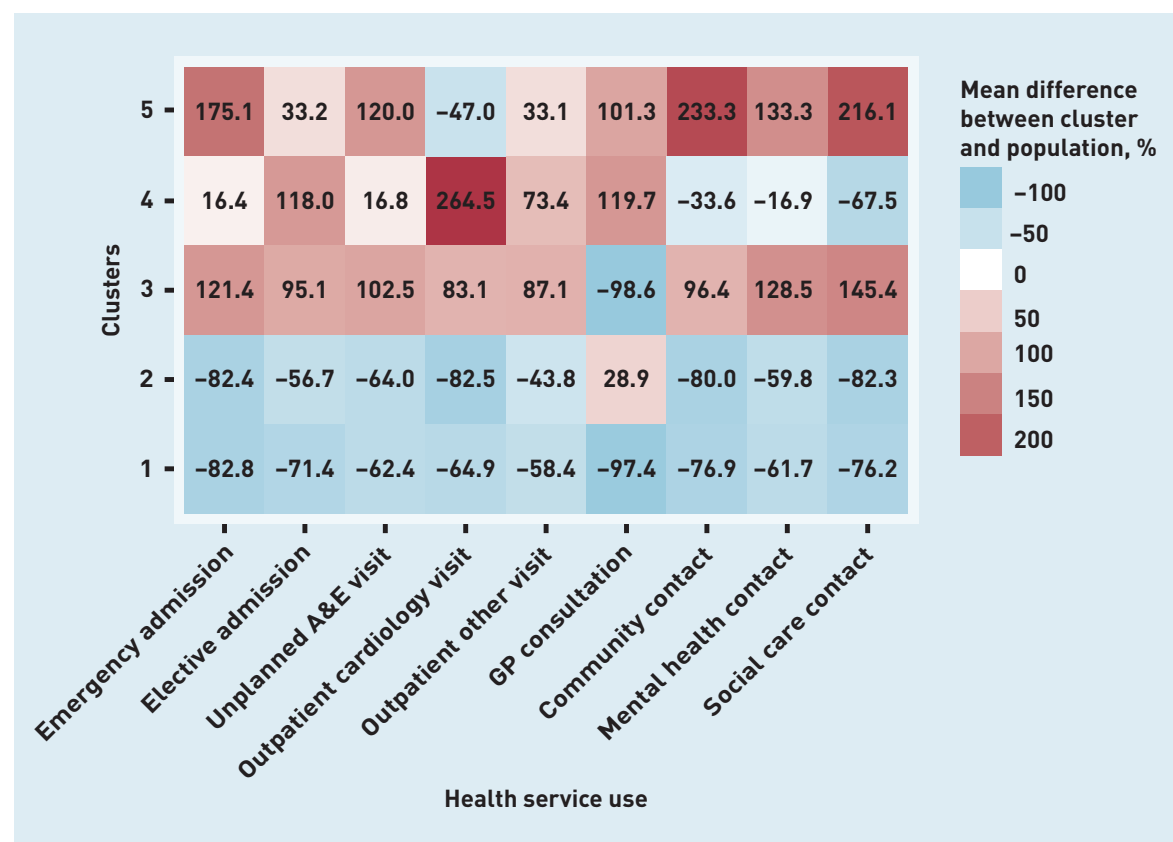

Figure 2. Heatmap of service utilisation by cluster: Numbers represent percentage difference between cluster mean and population mean values of each health utilisation variable.

$A \& E=$ accident and emergency. introduce bias; however, a transparent coding methodology to mitigate this was provided. Moreover, coded data rely on recorded information, meaning that certain diseases or service components may have been underestimated, for example, cardiac rehabilitation, or that certain primary care data coding may have been affected by payfor-performance schemes. The authors were unable to ascertain the reasons for the community care or mental health consultations as diagnosis coding was irregular. It is also likely that the fall in GP consultations during the study period was offset by more practice nurse contacts, which were not included in the dataset.

Lastly, though the dataset was based on adults living in a community setting from a large and ethnically diverse area in England, the findings may not be generalisable to the wider population of people with HF.

\section{Comparison with existing literature}

Few studies have attempted to quantify individual patterns of care in real-world settings beyond the hospital. Robertson et al described the burden of HF on the Australian healthcare system, but were only able to assess hospitalisation data, ${ }^{23}$ as was the case for the present authors previous work. ${ }^{24,25}$ Similarly, other studies have described a single dimensional aspect of health service use by the population of people with HF.26,27 The present findings are consistent with these, showing that secondary care use is high, ${ }^{23}$ participation in cardiac rehabilitation in the community is low, ${ }^{26,28}$ and that requirement for personal care, such as nursing and homecare services, is relatively common. ${ }^{27}$

An increase over time in most healthcare services use was observed in the present study, especially outpatient visits, but a surprising decrease of 11\% in GP consultations. Furthermore, only $60 \%$ of patients had GP appointments during the study period, which contrasts with the national GP Patient Survey of 2019,29 where $85 \%$ of patients reported having had a GP appointment in the past year. Potential explanations include the increasing workload and workforce pressures on primary care, changes in primary care practice with more frequent contacts with practice nurses and allied health professionals, a significant problem of access to care, and/or differences in case mix.

Another surprising finding is the apparently limited uptake of community cardiac rehabilitation. The National Audit of Cardiac Rehabilitation 2018 report ${ }^{28}$ suggests that around half of eligible patients take up cardiac rehabilitation. The report did indicate significant regional variation. However, it seems likely that differences in coding of data are responsible for the very low uptake in this analysis; 'rehabilitation' events may be recorded elsewhere and currently unavailable in WSIC, and 'community cardiology' may also include HF nurse domiciliary care.

The authors further report low use of mental health and social care services by patients with HF, but whether this observed level is appropriate is unclear without further assessment.

\section{Implications for research and practice}

The present finding of increased secondary and urgent care service use, low GP appointment use, and apparently limited cardiac rehabilitation is of concern and suggests a lack of multidisciplinary HF care. National Institute for Health and Care Excellence guidelines recommend an MDT approach, but there is no standard definition besides who should be involved and what should be achieved. " Each local area has unique challenges and requires tailored solutions; research is needed to establish the nature, location, timing, and intensity of the support needed by patients with HF. In an ethnically diverse area with a relatively young population like NWL, where deprivation level and ethnicity may affect a person's access to health care, creating a strong MDT embedded in primary care may be very pertinent. For example, practice nurses may target recently diagnosed 


\section{Funding}

The Dr Foster Unit at Imperial College London is partially funded by a grant from Dr Foster, a private healthcare information company. It is also partly funded by research grants from the National Institute for Health Research (NIHR) Health Services and Delivery Research (HS\&DR) (ref: 17/99/72). Martin R Cowie's salary is supported by the NIHR Cardiovascular Biomedical Research Unit at the Royal Brompton Hospital, London. None of the funders had any role in the conception, design, analysis, or reporting of this study

\section{Ethical approval}

Whole Systems Integrated Care (WSIC) is a dataset of North West London (NWL) residents who have consented to the anonymous data in their online health records for research purposes. Additionally, this specific study was approved by the Discover Research Advisory Group (DRAG), which is a nominated body that provides a governance mechanism for evaluating project applications requesting the WSIC de-identified dataset.

\section{Provenance}

Freely submitted; externally peer reviewed.

\section{Competing interests}

Alex Bottle, Dani Kim, and Paul Aylin had financial support through a research grant from Dr Foster for the submitted work. There were no financial relationships with any organisations that might have an interest in the submitted work in the previous 3 years and no other relationships or activities that could appear to have influenced the submitted work. Benedict Hayhoe is a GP working in the NHS.

\section{Acknowledgements}

The Department of Primary Care and Public Health at Imperial College London is grateful for support from the NWL NIHR Applied Research Collaboration and the Imperial NIHR Biomedical Research Centre.

\section{Discuss this article}

Contribute and read comments about this article: bjgp.org/letters

patients in primary care, that is, younger patients with fewer comorbidities, on early education and management, which may include additional telephone and/or specialist community support for those with lower socioeconomic status. This, in conjunction with hospital-based solutions, like early supported discharge plans for older patients, who are the highest users of secondary care, may provide significant and long-term benefits for the NWL area. Local solutions like these have been shown not only to reduce utilisation of health services but also to improve patient wellbeing and result in large cost savings for the NHS.30

Though the present data could not establish whether an MDT approach was implemented in the NWL area, it may well be that MDTs exist but their solutions are not translating into reduced secondary care use. Successful MDTs will require cooperation, coordination, and communication across health services. Reasons for ineffective multidisciplinary care could be posited through the following questions: is there an overarching coordinating unit for multidisciplinary care? Are the IT systems compatible for such care? Is information exchange readily available and safe? Is communication across settings both smooth and frequent? Is the approach sustainable? These questions illustrate how successful solutions will require sustained financial investments and the solid backing of all relevant stakeholders, and the sheer challenge of this may explain why many MDTs have had only neutral effects. ${ }^{2}$ 


\section{REFERENCES}

1. National Institute for Health and Care Excellence. Chronic heart failure in adults: diagnosis and management. NG106. 2018. https://www.nice.org.uk/ guidance/ng106 laccessed 19 May 2020).

2. Morton G, Masters J, Cowburn PJ. Multidisciplinary team approach to heart failure management. Heart 2018; 104(16): 1376-1382.

3. Legislation.gov.uk. Health and Social Care Act 2012. http://wnw.legislation.gov uk/ukpga/2012/7/contents/enacted (accessed 19 May 2020).

4. National Collaboration for Integrated Care and Support. Integrated care and support: our shared commitment. 2013. assets.publishing.service.gov. uk/government/uploads/system/uploads/attachment_data/file/198748/ DEFINITIVE_FINAL_VERSION_Integrated_Care_and_Support_-_Our_Shared_ Commitment_2013-05-13.pdf (accessed 19 May 2020).

5. Department of Health Cardiovascular Disease Team. Cardiovascular disease outcomes strategy: improving outcomes for people with or at risk of cardiovascular disease. 2013. assets.publishing.service.gov.uk/government/ uploads/system/uploads/attachment data/file/217118/9387-2900853-CVDOutcomes_web1.pdf (accessed 19 May 2020).

6. NHS England. Five year forward view. 2014. www.england.nhs.uk/wp-content/ uploads/2014/10/5yfv-web.pdf laccessed 19 May 2020).

7. Ponikowski P, Voors AA, Ankerk SD, et al. 2016 ESC guidelines for the diagnosis and treatment of acute and chronic heart failure: the task force for the diagnosis and treatment of acute and chronic heart failure of the European Society of Cardiology (ESC) developed with the special contribution of the Heart Failure Association (HFA) of the ESC. Eur Heart J 2016; 37(27): 2129-2200.

8. Yancy CW, Jessup M, Bozkurt B, et al. Writing Committee Members, American College of Cardiology Foundation/American Heart Association Task Force on Practice Guidelines. 2013 ACCF/AHA guideline for the management of heart failure: a report of the American College of Cardiology Foundation/American Heart Association Task Force on practice guidelines. Circulation 2013; 128(16): e240-e327.

9. Imperial College Health Partners, NHS. About Discover. https://uww. registerfordiscover.org.uk/about-discover/overview laccessed 19 May 2020).

10. NHS North West London Collaboration of Clinical Commissioning Groups. About us. https://www.healthiernorthwestlondon.nhs.uk/about/about laccessed 19 May 2020).

11. Herrett E, Gallagher AM, Bhaskaran K, et al. Data resource profile: Clinical Practice Research Datalink (CPRD). Int J Epidemiol 2015; 44(3): 827-836.

12. Lewis JD, Schinnar R, Bilker WB, et al. Validation studies of The Health Improvement Network (THIN) database for pharmacoepidemiology research. Pharmacoepidemiol Drug Saf 2007; 16(4): 393-401.

13. Ministry of Housing, Communities and Local Government. English indices of deprivation 2015. http://imd-by-postcode.opendatacommunities.org/imd/2015 laccessed 19 May 2020).

14. Khan NF, Perera R, Harper S, Rose PW. Adaptation and validation of the Charlson index for Read/OXMIS coded databases. BMC Fam Pract 2010; 11: 1

15. Windgassen S, Moss-Morris R, Goldsmith K, Chalder T. The importance of cluster analysis for enhancing clinical practice: an example from irritable bowel syndrome. J Ment Health 2018; 27(2): 94-96.
16. The Comprehensive R Archive Network. eulerr. https://cran.r-project.org/web/ packages/eulerr/readme/README.html (accessed 19 May 2020).

17. Szczepura A. Access to health care for ethnic minority populations. Postgrad Med J 2005; 81(953): 141-147.

18. M Morteruel, Rodriguez-Alvarez E, Martin U, Bacigalupe A. Inequalities in health services usage in a National Health System scheme: the case of a southern social European region. Nurs Res 2018; 67(1): 26-34.

19. Doherty E, O'Neill C. Estimating the health-care usage associated with osteoarthritis and rheumatoid arthritis in an older adult population in Ireland. $J$ Public Health 2014; 36(3): 504-510.

20. König $\mathrm{H}-\mathrm{H}$, Lehnert $\mathrm{T}$, Brenner $\mathrm{H}$, et al. Health service use and costs associated with excess weight in older adults in Germany. Age Ageing 2015; 44(4): 616 623.

21. Cheung JTK, Yu R, Wu Z, et al. Geriatric syndromes, multimorbidity, and disability overlap and increase healthcare use among older Chinese. BMC Geriatr 2018; 18(1): 147.

22. Jordan K, Porcheret M, Croft P. Quality of morbidity coding in general practice computerized medical records: a systematic review. Fam Pract 2004; 21(4): 396-412.

23. Robertson J, McElduff P. Pearson S-A, et al. The health services burden of heart failure: an analysis using linked population health data-sets. BMC Health Serv Res 2012; 12: 103

24. Bottle A, Goudie R, Bell D, et al. Use of hospital services by age and comorbidity after an index heart failure admission in England: an observational study. BMJ Open 2016; 6(6): e010669

25. Bottle A, Honeyford K, Chowdhury F, et al. Factors associated with hospital emergency readmission and mortality rates in patients with heart failure or chronic obstructive pulmonary disease: a national observational study. Southampton: National Institute for Health Research Journals Library, 2018.

26. Park LG, Schopfer DW, Zhang N, et al. Participation in cardiac rehabilitation among patients with heart failure. J Card Fail 2017; 23(5): 427-431.

27. Foebel AD, Hirdes JP, Heckman GA, et al. A profile of older communitydwelling home care clients with heart failure in Ontario. Chronic Dis Can 2011; 31(2): 49-57.

28. British Heart Foundation. The National Audit of Cardiac Rehabilitation (NACR) quality and outcomes report 2018. 2018. https://www.bhf.org. uk/informationsupport/publications/statistics/national-audit-of-cardiac rehabilitation-quality-and-outcomes-report-2018 (accessed 19 May 2020).

29. GP Patient Survey. Surveys and reports. 2019. https://www.gp-patient.co.uk/ surveysandreports (accessed 19 May 2020).

30. British Heart Foundation. Integrated Care: best practice. 2016. https://www.bhf. org.uk/informationsupport/publications/healthcare-and-innovations/integratedcare-best-practice (accessed 19 May 2020). 